\title{
AMORPHIZATION AND RECRYSTALLIZATION IN MeV ION IMPLANTED InP CRYSTALS ${ }^{\dagger}$
}

Fulin Xiong, C. W. Nieh, D. N. Jamieson, T. Vreeland, Jr., and T. A. Tombrello Materials Research Group, California Institute of Technology, Pasadena, California 91125.

\section{ABSTRACT}

A comprehensive study of $\mathrm{MeV}^{-15} \mathrm{~N}^{-}$-ion-implanted $\mathrm{InP}$ by a variety of analytical techniques has revealed the physical processes involved in $\mathrm{MeV}$ ion implantation into III-V compound semiconductors as well as the influence of post-implantation annealing. It provides a coherent picture of implant distribution, structural transition, crystalline damage, and lattice strain in InP crystals induced by ion implantation and thermal annealing. The experimental results from the different measurements are summarized in this report. Mechanisms of amorphization by implantation and recrystallization through annealing in $\mathrm{MeV}$-ion-implanted $\mathrm{InP}$ are proposed and discussed in light of the results obtained.

\section{INTRODUCTION}

Currently there is a considerable interest in $\mathrm{MeV}$ ion implantation into III-V compound semiconductors due to its potential to form buried heterojunction layers and modify electrical and optical properties as well as its use in 3-dimensional device processing. However, the extension of the ion implantation energy to the $\mathrm{MeV}$ range has raised many interesting questions about the mechanism of radiation-induced damage and its relation to implant distribution in crystalline solids. An understanding of the associated physical processes involved in $\mathrm{MeV}$ ion implantation as well as in the subsequent annealing is crucial before it can be universally applied. This emphasis is strongly modivated by the need of the microelectronic industry for new integrated circuit processing techniques and noval electronic device structure as well as optical laser and microwave devices. Work on GaAs can be found in many reports ${ }^{[1-3]}$, but there are few reports on $\operatorname{InP}^{[4-6]}$; however, most of the work concentrated on the use of $\mathrm{keV}$ ions.

Last year we reported ${ }^{[6]}$ the radiation-induced lattice contraction in $\mathrm{MeV}$ ion implanted InP through the $\mathrm{x}$-ray rocking curve studying. The effect observed was opposite to what was observed in $\mathrm{MeV}$-ion-implanted $\mathrm{GaAs}^{[2]}$. Further work has been carried out with $\mathrm{MeV}-\mathrm{N}^{+}$-ion implantation into InP crystals to investigate the physical processes involved, thus to obtain a better understanding of the mechanisms of amorphization and recrystallization that occures in $\mathrm{MeV}$ ion implantation and post-implantation annealing of III-V compound semiconductors. Subsequent characterization by a variety of analytical techniques has been undertaken. Nuclear resonant reaction analysis (NRRA) was employed to profile the implant depth distribution. The $\mathrm{x}$-ray rocking curve technique (XRC) was utilized to measure the radiation-induced lattice strain and damage. Channeling Rutherford backscattering spectrometry (CRBS) was conducted to profile the implantation-induced crystal damage and defect distribution. Cross sectional and high resolution transmission electron microscopy (XTEM and HRTEM) were performed to observe visually the structural phase transition and defect distribution. These results are summarized in this paper; the detailed description has been reported elsewhere ${ }^{[7]}$. A spike model to describe the mechanism of amorphization by ion implantation is proposed, along with phenomenological discussion of the mechanism for recrystallization through annealing in $\mathrm{MeV}$-ion-implanted InP.

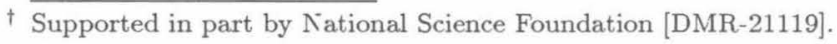




\section{AMORPHIZATION BY ION IMPLANTATION}

The detailed experimental procedure of ion implantation has been presented in the previous report ${ }^{[7]}$. InP(100) single crystal wafers were used throughout this study; they were n-type with a Sn-doping concentration of $7 \times 10^{16} / \mathrm{cm}^{3}$. In view of our interest in forming a buried semi-insulating layer and making simple the depth profiling of the implant distribution, $5 \mathrm{MeV}^{15} \mathrm{~N}^{-}$ions generated on the EN-tandem accelerator were used and uniformly projected onto the target (held at nominal room temperature) through a $6 \times 6 \mathrm{~mm}^{2}$ collimator with a flux less than $1 \mu \mathrm{A} / \mathrm{cm}^{2}$. The as-implanted samples were subsequently characterized by the analytical techniques mentioned above.

The depth profiles observed by NRRA show that the $5-\mathrm{MeV}_{-1}{ }^{15} \mathrm{~N}$-ions implanted into InP are distributed in a well-defined Gaussian shape with the center at a depth of $3.71 \mu \mathrm{m}$ and a width of $0.60 \mu \mathrm{m}^{[7]}$. These distributions are dose-independent and are uniquely determined by the incident energy of the ions.

The process of amorphization by $\mathrm{MeV}$ ion implantation has been clearly revealed by the combination of CRBS and XTEM. As is illustrated in the Figure 1a for a set of CRBS spectra from as-implanted samples, the ${ }^{4} \mathrm{He}$ particles suffer more and more de-channeling as they go deeper into the sample. As the implant dose is increased, the de-channeling yield dramatically increases close to the random until the saturation is reached. Then, saturation extends gradually towards the sample surface with increasing dose, and at a dose of $1 \times 10^{16} / \mathrm{cm}^{2}$ the saturation has approached the surface. (The spectrum is not shown as it looks nearly same as for the random, except a slight drop in a few channels near the surface.) Figures 2abc show three conventional XTEM micrographs from asimplanted samples with different implant doses. The sample surface lies at the left-hand side. The regions with irregular dark spots are found to be heavily damaged with a high density of dislocations and defect centers. The strong contrast, featureless bands illustrate the buried amorphous layers. Summarizing the results of CRBS and XTEM, one can conclude that the de-channeling yield spectra in CRBS provides a picture of the depth distribution of the radiation-induced atomic displacement and damage in InP crystals. The saturation indicates the formation of an amorphous layer in the sample by implantation. It was found that at doses below $5 \times 10^{14} / \mathrm{cm}^{2}$ a high density of radiation damage was induced only in the region near the end of ion range, while on the samples implanted with doses over $1 \times 10^{15} / \mathrm{cm}^{2}$ the amorphous layers formed with their inner interface at a depth of about $4 \mu \mathrm{m}$, where the concentration of implanted ${ }^{15} \mathrm{~N}^{-}$was at its half maximum. It has also been shown that the width of the buried layer extends towards the surface with increasing implant dose, while the inner interface depth is almost fixed, independent of the dose.

More detailed characteristics of the structural changes caused by implantation have been observed through high resolution XTEM analysis. Figure 3 presents lattice image micrographs from as-implanted samples. Fig 3a shows a high density of defect clusters induced by implantation at low dose $\left(5 \times 10^{14} / \mathrm{cm}^{2}\right)$, and Fig $3 \mathrm{~b}$ illustrates the sharp amorphous/crystalline interface in a high-dose-implanted sample $\left(1 \times 10^{16} / \mathrm{cm}^{2}\right)$. A low density of microtwins which appeared on the substrate crystal side along $\{111\}$ directions may have been induced through the channeling effect or in-situ thermal annealing effect during implantation.

The phenomenon of amorphization in InP by ion implantation has been also observed with the XRC technique. The results, as shown in Figure 4, illustrate changes of implantation-induced strain with increasing the implant dose. The strain saturation occurs at a dose higher than $1 \times 10^{15} / \mathrm{cm}^{2}$ to a perpendicular strain level of $-0.060 \pm 0.002 \%$, which is equal to the case of $15-\mathrm{MeV}-\mathrm{Cl}$-ion-implanted $\operatorname{InP}(100)^{[6]}$. This saturation is believed to be an indication of amorphization occured inside the crystal near the end of ion 

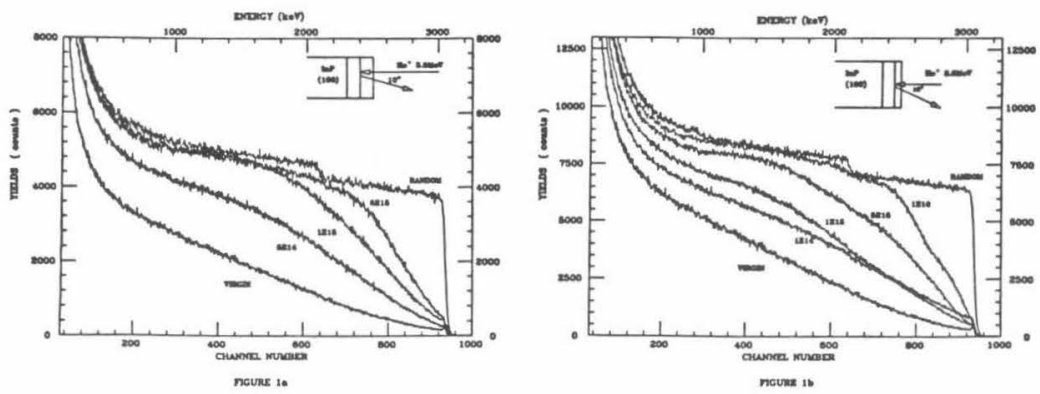

Fig. 1. Channeling RBS spectra of InP crystals implanted with $5 \mathrm{MeV}{ }^{15} \mathrm{~N}$ at room temperature with a sequence of doses as marked beside the curves, (1a.) as-implanted, (1b.) annealed. See details in the text.
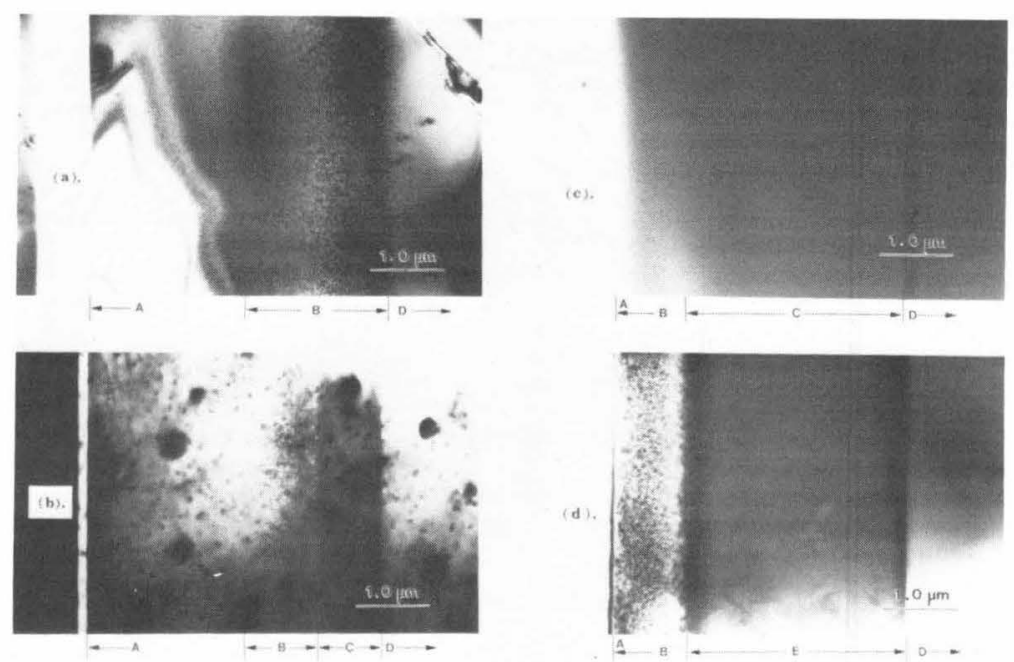

Fig. 2. XTEM micrographs of ${ }^{15} \mathrm{~N}$ ion implanted $\mathrm{InP}$ specimens on the entired implanted regions with different doses: (a) $5 \times 10^{14} / \mathrm{cm}^{2}$, as-implanted, (b) $1 \times 10^{15} / \mathrm{cm}^{2}$, as-implanted, (c) $1 \times 10^{16} / \mathrm{cm}^{2}$, as-implanted, and (d) $1 \times 10^{16} / \mathrm{cm}^{2}$, annealed. As marked in the figure, A is the surface region; B, heavily damaged region; C, amorphous region; D, substrate crystal; and $E$, recrystallized region. See details in the text. 

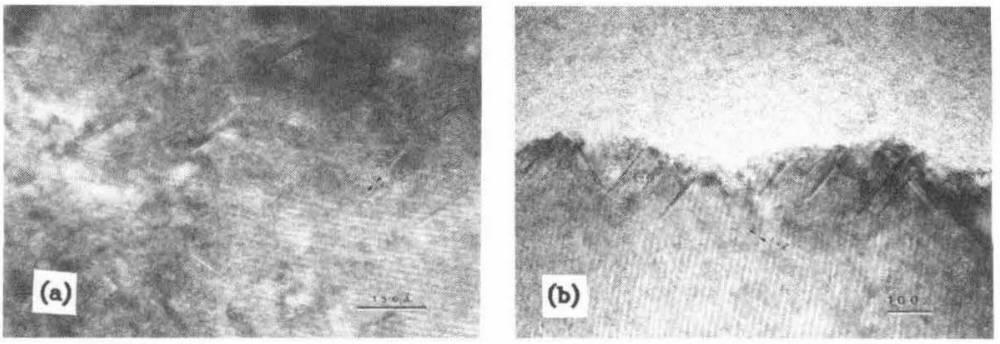

Fig. 3. HRTEM micrographs of as-implanted samples: (A) low dose $\left(5 \times 10^{14} / \mathrm{cm}^{2}\right)$, radiationinduced damage with a high density of implantation-induced defects ( stacking faults and dislocation loops ) is shown; (B) high dose $\left(1 \times 10^{16} / \mathrm{cm}^{2}\right)$, a buried sharp interface between the implantation-induced amorphous zone and the crystalline substrate is presented.
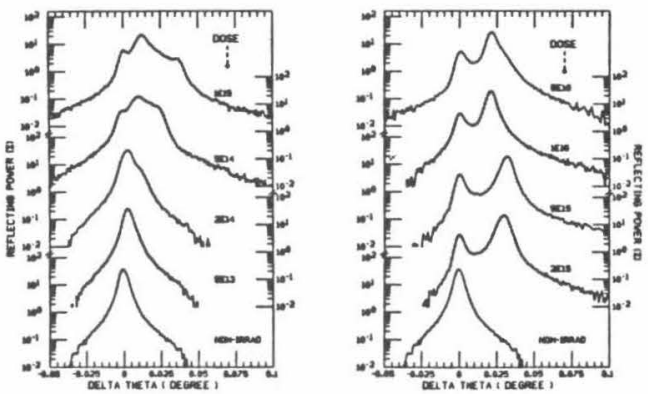

Fig. 4.

$\mathrm{X}$-ray rocking curves of the $5 \mathrm{MeV}-{ }^{15} \mathrm{~N}$ ion-implanted InP from (400) symmetrical diffraction with respect to the InP $(100)$ substrate. The radiation-induced strain and the strain saturation are shown. See details in the text.
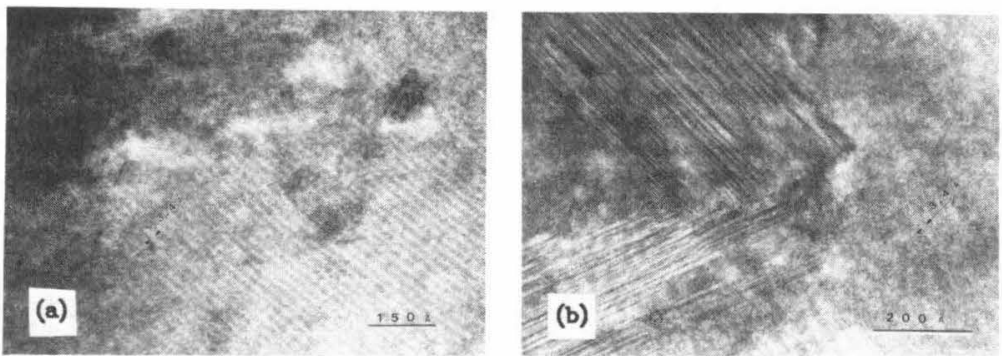

Fig. 5. HRTEM micrographs of post-implantation annealed samples: (A) low dose $\left(5 \times 10^{14} / \mathrm{cm}^{2}\right)$, the defect density has decreased dramatically, comparing to the case shown in Fig. 3a. Only a few stacking faults perferentially microfaceted along $\{111\}$ directions. (B) high dose $\left(1 \times 10^{16} / \mathrm{cm}^{2}\right)$, a thermal annealing structure formed through recrystallization in the original implantation-induced amorphous-crystalline interface is illustrated. A high density of long range microtwin clusters and stacking fault boundles appears along $\{111\}$ directions ( comparablly see Fig. 3b). 
range, as was established by CRBS and XTEM results presented (also see the discussion section).

\section{RECRYSTALLIZATION THROUGH THERMAL ANNEALING}

Post-implantation thermal annealing was performed in a graphite strip heater with a $\mathrm{H}_{2}$ gas flow at a $500 \mathrm{C}$ temperature or 20 minutes. Isochronal annealing was carried out in 50C degree step size for 15 minutes for XRC study. The sample was arranged in the face-to-face configuration with a virgin crystal which provides a local phorsphorus presure to prevent sample surface dissociation.

After high temperature annealing, no redistribution of ${ }^{15} \mathrm{~N}^{-}$has been found by NRRA within its detection limit. This implies that migration and thermal diffusion of implanted ${ }^{15} \mathrm{~N}^{-}$in the implanted $\mathrm{InP}$ are not significant. However, through thermal annealing structural re-ordering in the damaged regions and recrystallization in the buried amorphous layers have taken place. The XRC study on isochronically-annealed samples has given a result very similar to that in the early report ${ }^{[6]}$ on the work of $\mathrm{MeV}-\mathrm{Cl}$ implanted $\operatorname{InP}(100)$. Gradual strain recovery was observed through annealing with a jump stage at $250 \mathrm{C}-350 \mathrm{C}$; complete recovery of lattice strain occured at $450 \mathrm{C}$. A wellshaped peak from the damage-recovered surface and the substrate appeared at the exact position as the peak from the undamaged crystal, but the reflecting intensity dropped to about half magnitude and the width was broadened. This is believed to be due to the absorption and scattering by the buried disordered layer deep inside the crystal.

Dechanneling features in CRBS analysis are illustrated in Figure 1b. Yields in the original sample damaged regions have been dramatically reduced (See spectrum for the dose $5 \times 10^{14} / \mathrm{cm}^{2}$ and others in the regions near the surface), while yields from the original buried amorphous regions remain as high as the random. These indicate the recovery of the surface damage and defects and the formation of highly disordered crystralline structures inside through annealing. These have been confirmed by XTEM on the annealed samples as shown, for example, in Fig $2 \mathrm{~d}$ for an implanted dose of $1 \times 10^{16} / \mathrm{cm}^{2}$. The surface damaged region was annealed, and the point defect density was significantly reduced. Nucleation has taken place in the center of the buried amorphous layer to form the polycrystalline structure in the center part. Solid phase epitaxial regrowth from two crystalline-amorphous interfaces were stimulated towards the center to form highly disordered structures within the buried band near the interfaces. These fine structural features have been observed in the HRTEM presented in Figure 5. Fig 5a shows the reduction of defect density in a low-dose-implanted and annealed sample (comparing with Fig 4a). In Fig 5b the inner interface structure in a high-dose-implanted and annealed sample is shown. High density of long range microtwin clusters and stacking fault bundles grow along $\{111\}$ directions, seeding from defects originated during implantation on the amorphous-crystalline interface (see Fig 4b).

\section{MECHANISMS - - - Spike Damage and Epitaxial Regrowth}

The processes of structural transition of implantation-induced amorphization and thermally activated recrystallization in $\mathrm{MeV}$-ion-implanted III-V compound semiconductors are complex. They are quite different from those of $\mathrm{Si}$, since primary defect formation in a binary compound is much more complicated and the threshold energy for damage production and crystal regrowth is lower. Various models have been proposed to describe the crystalline-amorphous transition in ion-implanted semiconductor crystals, such as early studies by Seitz ${ }^{[8]}$, Gonser and Okkerse ${ }^{[9]}$ and most recently reviewed by Sadana ${ }^{[5]}$.

Based upon the results presented above we propose the following model for the amorphization and recrystallization in $\mathrm{MeV}$-ion-implanted InP. First, we classify the effect of the ion-solid interaction in the crystal during $\mathrm{MeV}$ ion implantation into two major 
processes: electronic and nuclear spikes. The electronic spike is generated through electronic excitation and ionization of the target atoms by the high energy ions and causes electronic damage to the crystal in the region near the surface, where the incident ion energy loss is dominated by this process. The nuclear spike is produced in the low energy range just before the ions stop due to nuclear collisions, through which the crystal suffers direct displacemental damage. Due to the spike effect, the nuclear cascade collision may locally generate a high temperature along the ion transport track near the end of ion range. When the instanteous ion energy loss is intense, this temperature can reach the InP melting point and the material locally melts, but is quickly quenched and the material resolidifies in the quasi-liquid (amorphous) form, which is different from the crystalline structure that would form if the cooling occurred more slowly. The presence of these small damaged regions gives rise to tension, the surrounded rigid framework of the undamage lattice prevents lattice relaxation, causing the lattice strain as observed by the XRC. As the implant dose increases, individual damage will spread and overlap until a continuous buried amorphous layer forms. The damage gradiently increases from the surface to its maximium range with a sharp interface at the inner boundary as shown in Fig. 3 b. The formation of the continuous amorphous layer provides an elastic region for the lattice strain to relax. The lattice strain saturation occurs when the strain production and relaxation reach an equilibrium stage. Thus, the strain saturation level is expected to be uniquely defined by the nature of the target crystal, independent of the ion species and incident energy. This is found in many cases for $\operatorname{InP}(100)$ as implanted by $15 \mathrm{MeV}^{35} \mathrm{Cl}$ ${ }^{[6]}, 8 \mathrm{MeV}^{-16} \mathrm{O}^{[10]}$, and $5 \mathrm{MeV}^{-15} \mathrm{~N}^{-[7]}$ which all produce the same perpendicular strain saturation level of $-0.060 \pm 0.002 \%$.

Recrystallization during high temperature annealing is also proposed to go through two kinds of processes: homogeneous nucleation and solid phase epitaxial regrowth. The first involves thermally-activated nucleation in the central transition region, beginning with formation of small crystal nuclei. As there are a large number of growth fronts in various orientations, there is a growth competition among neighboring crystallites, resulting in a polycrystalline structure in the center of the buried layer. The other process occurs at crystalline-amorphous interfaces. Atoms in the amorphous region make bonds with those in the single crystal substrate or the upper damaged crystal surface. The rough nature of the boundaries at the interfaces and regrowth orientation effect ${ }^{[1,5]}$ eventually result in a microfaceted and simply connected growth front from the interfaces. It would be expected that the growth rate in the [100] direction is fastest followed by [110], and then [111]. However, as predicted by Sadana et. al. ${ }^{[1,5]}$, the $\{111\}$ directions would have a high probability for nucleating planar microtwin defects, and the $\{111\}$ facets would provide favorable sites for accommodation of dopant concentration above the solid solubility limit and non-stoichiometry which is produced by the implantation. Seeding from the original stacking faults and twins formed during implantation in the crystalline substrate, bundles of stacking faults will propagate rapidly together with solid phase epitaxial regrowth. Moreover, there would be a competition between these two growth processes. The recrystallization will be end when epitaxial layers with stacking fault bundles from both interfaces impinges upon the central nucleated polycrystalline layer.

\section{CONCLUSION}

In conclusion, our study has demonstrated the complementary nature of the analytical capabilities of the techniques used for the investigation of the processes involved in $\mathrm{MeV}$ ion implantation, and also has clearly revealed substantial changes in structural properties, radiation-induced defect distribution and build-up with increasing implant dose in MeV-ion-implanted InP samples, as well as the influence of thermal annealing. 
These have led to a better understanding of the mechanisms of MeV-ion-implantationinduced amorphization and subsequent thermal annealing stimulated recrystallization in InP compound semiconductors. The kinetic energy of the implanted ions defines a maximium depth of the buried layer. The ion-solid interaction induced electronic and nuclear spikes cause electronic damage in the region near the target surface and structural damage in the region near the end of the ion stop range. When the implant dose increases over $1 \times 10^{15} / \mathrm{cm}^{2}$, amorphization occurs inside. The buried amorphous layer extends towards the surface as the implant dose increases. During high temperature annealing, thermallyactivated recrystallization in the buried amorphous layer is stimulated. In the center of the buried layer, nucleation takes place to form a polycrystalline strucure. Near the amorphous-crystalline interfaces, solid phase epitaxial regrowth emerges. Seeding from the implantation-induced defects at the interfaces, a highly disordered structure with long range stacking faults and microtwins grows, impinging upon the central polycrystalline structure.

It is noted here that electrical characterization of $\mathrm{MeV}-\mathrm{N}$-ion-implanted $\operatorname{InP}(100)$ has been also carried out, which indicates that a buried semi-insulating layer may be formed by high dose nitrogen ion implantation, which can be used for fabricating optical laser devices. This result and its application to device fabrication will reported later ${ }^{[11]}$.

\section{ACKNOWLEDGEMENTS}

The authors greatly acknowledge Prof. M-A. Nicolet for his valuable discussion on the CRBS analysis, and Carol Garland for her assitance on the TEM work.

\section{REFERENCES}

1. D. K. Sadana, T. Sands, and J. Washburn, Appl. Phys. Lett. 44(6) (1984) 623.

2. C. R. Wie, T. Vreeland, Jr. and T. A. Tombrello, Nucl. Inst. and Meth. $\underline{B 16}$ (1986) 44; Phys. Rev. B33 (1986) 4083; J. Appl. Phys. $\underline{59}$ (1986) 3743.

3. T. T. Bardin, J. G. Pronko, F. A. Junga, W. A. Opyd, A. J. Mardinly, F. Xiong and T. A. Tombrello, Nucl. Instr. and Meth. B24/25 (1987) 548.

4. M. Slater, S. Kostic, M. J. Nobes and G. Carter, Nucl. Instr. and Meth., B7/8 (1985) 429.

5. D. K. Sadana, Nucl. Instr. and Meth., B7/8 (1985) 375, and references therein.

6. C. R. Wie, T. Jones, T. A. Tombrello, T. Vreeland, F. Xiong, Z. Zhou, G. Burns and F. H. Dacol, Mat. Res. Soc. Symp. Proc., Vol. 74 (1987) 517.

7. Fulin Xiong, C. W Nieh, T. A. Tombrello, D. N. Jemieson, and T. Vreeland, Jr., Proceeding of the International Symposium on Applications of Ion Beam Produced by Small Accelerators, Oct. 1987, Jinan, China.

8. F. Seitz, Physics Today, 5(6) (1952) 6.

9. C. Gonser and B. Okkerse, Phys. Rev. 109(3) (1958) 663.

10. F. Xiong, Tandem Lab Report, FX-01(1987), Caltech, unpublished.

11. F. Xiong, H. Wang, T. R. Chen, and T. A. Tombrello, to be published. 